\title{
An Area and Delay Efficient Csla Architecture
}

\author{
1. J. Pravin adlin , ${ }^{2 .}$ C. Palaniappan \\ 1, 2. Department of Electronics and Communication Engineering \\ PG Scholar, Mount Zion College of Engineering \&Technology, Pudukkottai, India \\ Assistant Professor, Mount Zion College of Engineering \& Technology, Pudukkottai, India
}

\begin{abstract}
Carry select adder (CSLA) is known to be the fastest adder among the conventional adder structures. Due to the rapidly growing mobile industry not only the faster arithmetic unit but also less area and low power arithmetic units are needed. The modified CSLA architecture has developed using Binary to Excess-1 converter $(B E C)$. This paper proposes an efficient method which replaces the BEC using D latch. Experimental results are compared and the result analysis shows that the proposed architecture achieves the three folded advantages in terms of area, delay and power.
\end{abstract}

Index Terms- area efficient, CSLA, low power and BEC

\section{Introduction}

Area and power have major role in the designing of integrated circuit because of the increase in popularity of portable systems as well as the rapid growth of power density in VLSI circuits. Addition usually influences strongly on the overall performance of digital systems and a crucial arithmetic function. Adders are most widely used in electronic applications. For example, in microprocessors, millions of instructions per second are performed. Due to the increase in the portability of the devices like mobile, laptop etc. require more battery backup. Low power and area efficient addition and multiplication have always been a fundamental requirement of high performance processors and systems.

Designing efficient adder is the most difficult problem for researchers in VLSI design. The carry-select adder (CSLA) provides a compromise between small area but longer delay ripple carry adder (RCA) and larger area with shorter delay carry look-ahead adder [6]. CSLA uses multiple pairs of ripple carry adder (RCA) to generate partial sum and carry by considering carry input $\mathrm{Cin}=0$ and $\mathrm{Cin}=1$, then the final sum and carry are selected by multiplexers (mux) [3].

The modified CSLA using BEC $[2,4,6]$ has reduced area and power consumption with slight increase in delay. The basic idea of the proposed architecture is that which replaces the BEC by reducing the group. The proposed architecture reduces the area, delay and power.

\section{BINARY TO EXCESS-1 CONVERTOR (BEC)}

BEC is a circuit used to add 1 to the input numbers. A circuit of 3-bit BEC and the function table is shown in Fig 1.5 and Table 1 respectively. The main objective of this project is

to reduce the gate level by using Binary to Excess-1 Converter. In order to reduce the delay and power we use $\mathrm{n}+1$ Binary to Excess-1 Converter instead of $\mathrm{n}$ RCA.

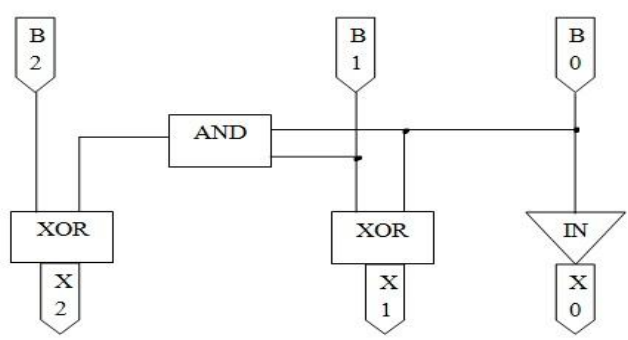

Fig.1 3-Bit Binary to Excess-1 Converter

Fig. 1 shows the basic function of the CSLA. One input for 6:3 mux is BEC output $\left(\mathrm{B}_{2}, \mathrm{~B}_{1}\right.$ and $\left.\mathrm{B}_{0}\right)$ and another input for the mux is the RCA with $\mathrm{C}_{\mathrm{in}}=0$. This produces the two possible partial results in parallel and the mux is used to select either the BEC output or the direct inputs according to the control signal $\mathrm{C}_{\mathrm{in}}$. The importance of the BEC logic is the large silicon area reduction. 
The Boolean expression of the 3-bit BEC are shown below:

$$
\begin{aligned}
& \mathrm{X}_{0}=\sim \mathrm{B}_{0} \\
& \mathrm{X}_{1}=\mathrm{B}_{0} \oplus \mathrm{B}_{1} \\
& \mathrm{X}_{2}=\mathrm{B}_{2} \oplus\left(\mathrm{B}_{1} \times \mathrm{B}_{0}\right)
\end{aligned}
$$

\begin{tabular}{|c|c|c|c|c|c|}
\hline \multicolumn{2}{|l|}{ BINARY [3:0] } & \multicolumn{3}{c|}{ EXCESS-1 [3:0] } \\
\hline B $_{\mathbf{2}}$ & $\mathbf{B}_{\mathbf{1}}$ & $\mathbf{B}_{\mathbf{0}}$ & $\mathbf{X}_{\mathbf{2}}$ & $\mathbf{X}_{\mathbf{1}}$ & $\mathbf{X}_{\mathbf{0}}$ \\
\hline 0 & 0 & 0 & 0 & 0 & 1 \\
\hline 0 & 0 & 1 & 0 & 1 & 0 \\
\hline 0 & 1 & 0 & 0 & 1 & 1 \\
\hline 0 & 1 & 1 & 1 & 0 & 0 \\
\hline 1 & 0 & 0 & 1 & 0 & 1 \\
\hline 1 & 0 & 1 & 1 & 1 & 0 \\
\hline 1 & 1 & 0 & 1 & 1 & 1 \\
\hline 1 & 1 & 1 & 0 & 0 & 0 \\
\hline
\end{tabular}

Table 1 Function Table of 3-Bit Binary to Excess-1 Convertor

\section{A. Delay And Area Calculation}

The AND, OR, and Inverter (AOI) implementation of an XOR gate is shown in Fig 2. The gates between the dotted lines are performing the operations in parallel and the numeric representation of each gate indicates the delay contributed by that gate.

The delay and area evaluation methodology considers all gates to be made up of AND, OR, and Inverter, each having delay equal to 1 unit and area equal to 1 unit. Then add up the number of gates in the longest path of a logic block that contributes to the maximum delay.

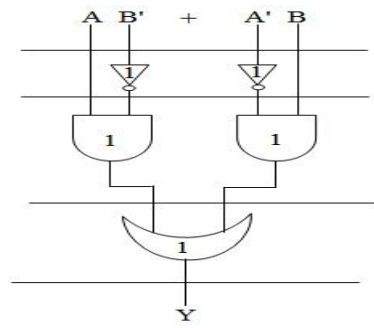

Fig.2 Delay and Area Evaluation of an XOR Gate

The area evaluation is done by counting the total number of AOI gates required for each logic block. The delay calculation is done by using the parallel performance of work in XOR gate.

Based on this approach, the CSLA adder blocks of 2:1 mux, Half Adder (HA), and Full Adder (FA) are evaluated and listed in Table 2

Table 2 Delay and Area count of the blocks of CSLA

\begin{tabular}{|c|c|c|}
\hline Adder Blocks & Delay & Area \\
\hline XOR & 3 & 5 \\
\hline $2: 1 \mathrm{Mux}$ & 3 & 4 \\
\hline Half Adder & 3 & 6 \\
\hline Full Adder & 6 & 13 \\
\hline
\end{tabular}




\section{Regular Carry Select Adder}

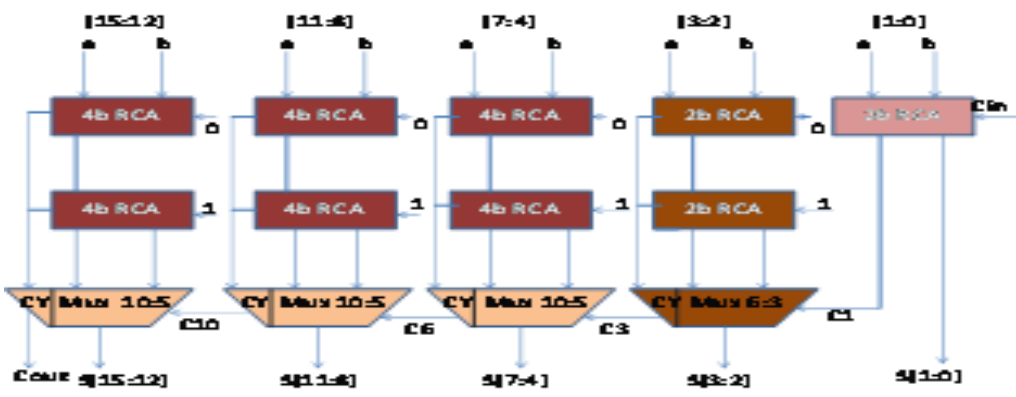

Fig.3.Architecture of regular CSLA

A 16-bit carry select adder can be developed in two different sizes namely uniform block size and variable block size. Similarly a 32, 64 and 128-bit can also be developed in two modes of different block sizes. Ripple-carry adders are the simplest and most compact full adders, but their performance is limited by a carry that must propagate from the least-significant bit to the most-significant bit. The various 16, 32, 64 and 128-bit CSLA can also be developed by using ripple carry adders. The speed of a carry-select adder can be improved upto $40 \%$ to $90 \%$, by performing the additions in parallel, and reducing the maximum carry delay.

Fig 3 shows the Regular structure of 64-bit SQRT CSLA. It includes many ripple carry adders of variable sizes which are divided into groups.

Group 0 contains 2-bit RCA which contains only one ripple carry adder which adds the input bits and the input carry and results to sum [1:0] and the carry out. The carry out of the Group 0 which acts as the selection input to mux which is in group 1, selects the result from the corresponding RCA (Cin=0) or RCA $(\mathrm{Cin}=1)$. Similarly the remaining groups will be selected depending on the Cout from the previous groups.

In Regular CSLA, there is only one RCA to perform the addition of the least significant bits [1:0]. The remaining bits (other than LSBs), the addition is performed by using two RCAs corresponding to the one assuming a carry-in of 0 , the other a carry-in of 1 within a group. In a group, there are two RCAs that receives the same data inputs but different Cin. The upper adder has a carry-in of 0 , the lower adder a carry-in of 1 . The actual Cin from the preceding sector selects one of the two RCAs. That is, as shown in the Fig.3, if the carry-in is 0 , the sum and carry-out of the upper RCA is selected, and if the carry-in is 1, the sum and carry-out of the lower RCA is selected.

For this Regular CSLA architecture, the implementation code, for the Full Adders and Multiplexers of different sizes $(6: 3,8: 4,10: 5$ up to $24: 11)$ were designed initially. The regular 64-bit, 128-bit CSLA were implemented by calling the ripple carry adders and all multiplexers.

\section{Modified Carry Select Adder}

The Binary to excess one Converter (BEC) replaces the ripple carry adder with $\mathrm{Cin}=1$, in order to reduce the area and power consumption of the regular CSLA. The modified16-bit CSLA using BEC is shown in Fig. 4 [1]. The structure is again divided into five groups with different bit size RCA and BEC. The group 2 of the modified 16-bit CSLA is shown Fig. 7. By manually counting the number of gates used for group 2 is 43 (full adder, half adder, multiplexer, BEC).

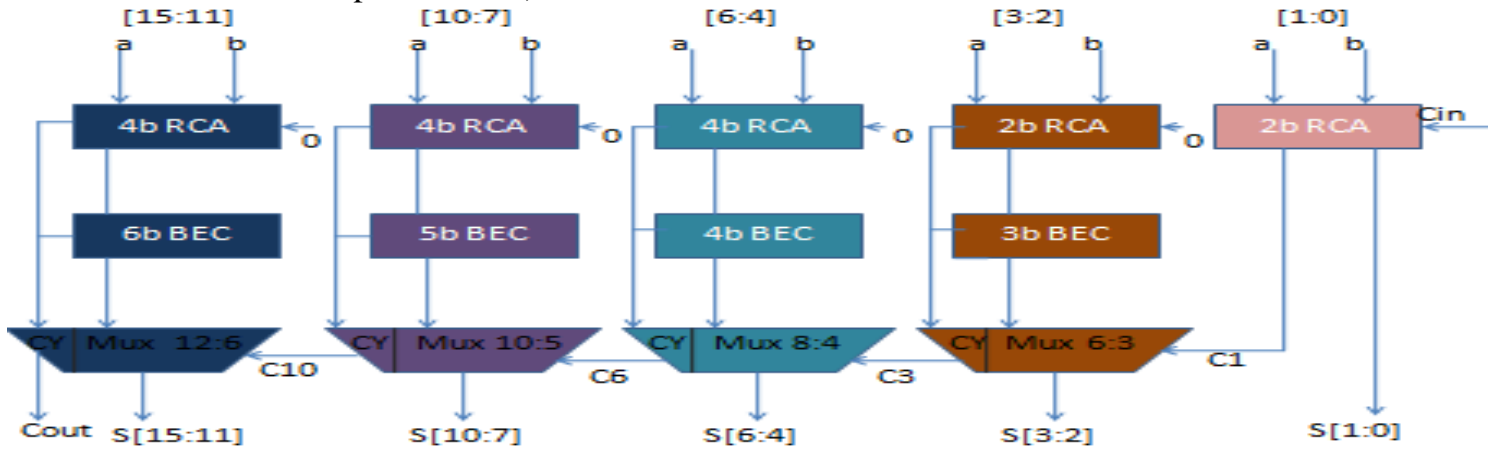

Fig.4.Modified Carry Select Adder 
One input to the mux goes from the RCA with $\mathrm{Cin}=0$ and other input from the BEC. Comparing the group 2 of both regular and modified CSLA, it is clear that BEC structure reduces the area and power. But the disadvantage of BEC method is that the delay is increasing than the regular CSLA [8].

\section{Proposed Carry Select Adder}

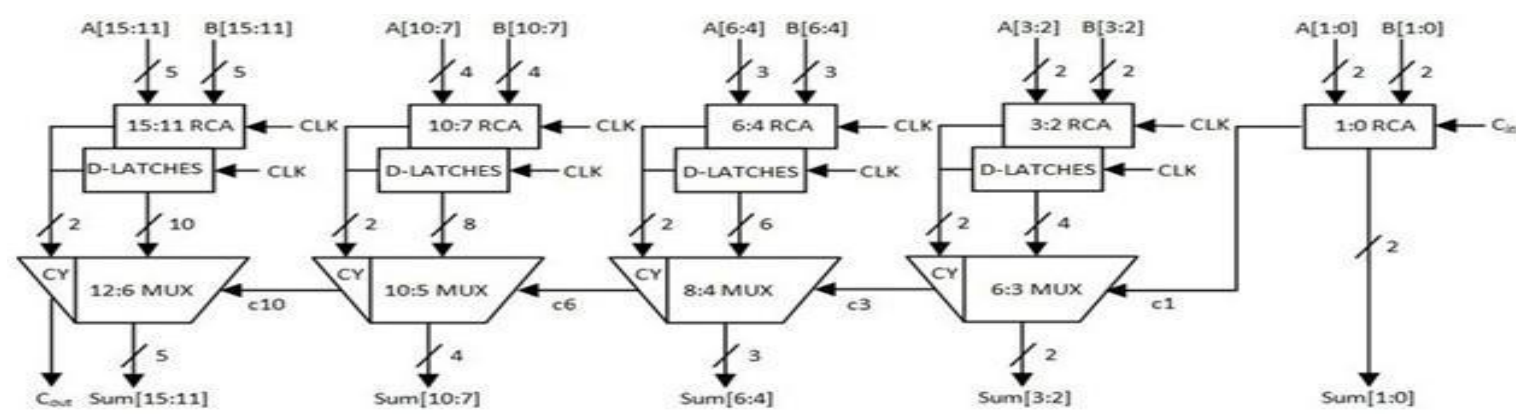

Fig.5.Proposed Carry Select Adder

This method replaces the BEC add one circuit by D-latch with enable signal. Latches are used to store one bit information. Their outputs are constantly affected by their inputs as long as the enable signal is asserted. In other words, when they are enabled, their content changes immediately according to their inputs.

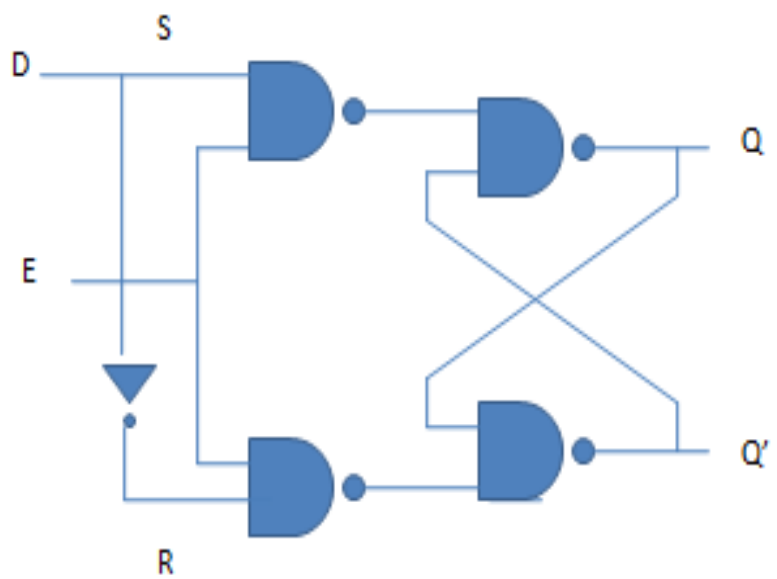

Fig.6.D Latch

This is 16-bit adder in which least significant bit (LSB) adder is ripple carry adder, which is 2 bit wide. The upper half of the adder i.e, most significant part is 14-bit wide which works according to the clock. Whenver clock goes high addition for carry input one is performed. When clock goes low then carrry input is assumed as zero and sum is stored in adder itself. it can understand that latch is used to store the sum and carry for $\mathrm{Cin}=1$.

Carry out from the previous stage i.e, least significant bit adder is used as control signal for multiplexer to select final output carry and sum of the 16-bit adder. If the actual carry input is one, then computed sum and carry latch is accessed and for carry input zero MSB adder is accessed. Cout is the output carry.

The internal structure of group 2 of the proposed 16-bit CSLA. The group 2 performed the two bit addition which are a2 with b2 and a3 with b3. This is done by two full adder (FA) named FA2 and FA3 respectively. The third input to the full adder FA2 is the clock instead of the carry and the third input to the full adder FA3 is the carry output from FA2. The group 2 structure has three D-Latches in which two are used for store the sum 2 and sum 3 from FA2 and FA3 respectively and the last one is used to store carrry. Multiplexer is used for selecting the actual sum and carry according to the carrry is coming from the previous stage. The 6:3 multiplexer is the combination of 2:1 multiplexer. 


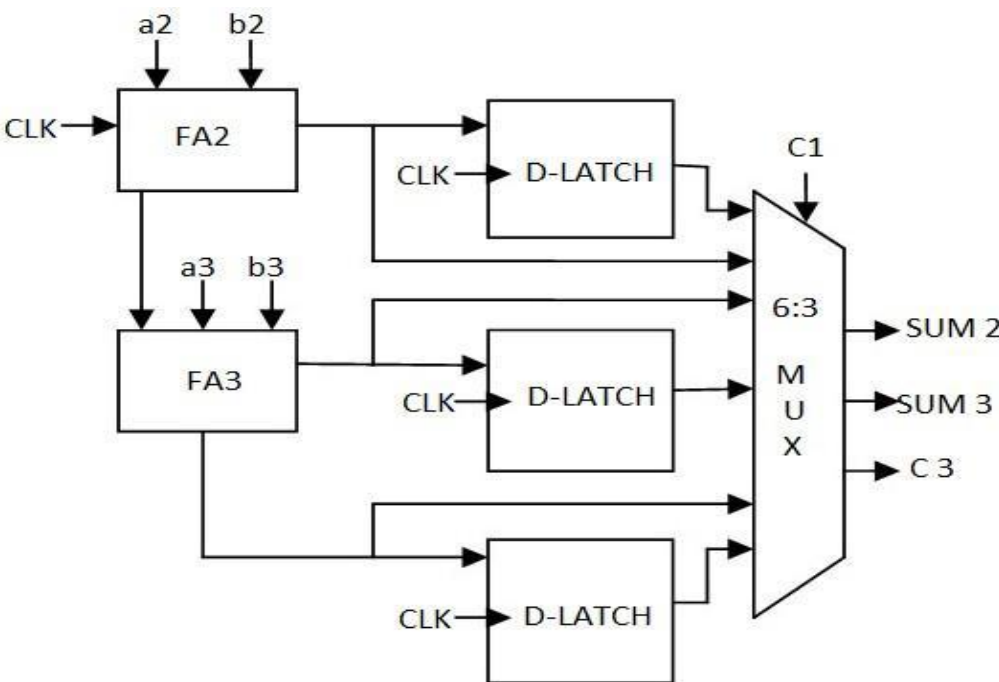

Fig.7.Group 2 Structure

When the clock is low a2 and b2 are added with carry is equal to zero. Because of low clock, the DLatch is not enabled. When the clock is high, the addition is performed with carry is equal to one. All the DLatches are enabled and store the sum and carry for carry is equal to one. According to the value of c1 whether it is 0 or 1 , the multiplexer selected the actual sum and carry.

\section{Comparision Results}

\begin{tabular}{|l|l|l|l|}
\hline $\begin{array}{l}\text { WORD } \\
\text { SREE }\end{array}$ & ADDER & DELAY(ns) & AREA(no of gate) \\
\hline 8 bit & Regular & 14.26 & 192 \\
\hline & Modified & 16.06 & 162 \\
\hline & Proposed & 10.725 & 14 \\
\hline 16 bit & Regular & 17 & 438 \\
\hline & Modified & 21.35 & 384 \\
\hline & Proposed & 13.583 & 28 \\
\hline 32 bit & Regular & 27.97 & 870 \\
\hline & Modified & 35.44 & 762 \\
\hline & Proposed & 16.511 & 60 \\
\hline 64 bit & Regular & 53.82 & 1698 \\
\hline & Modified & 63.61 & 1518 \\
\hline & Proposed & 23.511 & 118 \\
\hline
\end{tabular}

\section{Experimental Results}

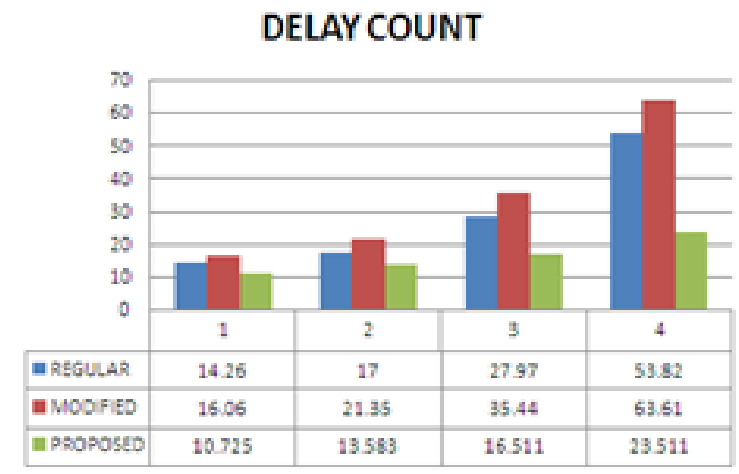




\section{AREA COUNT}

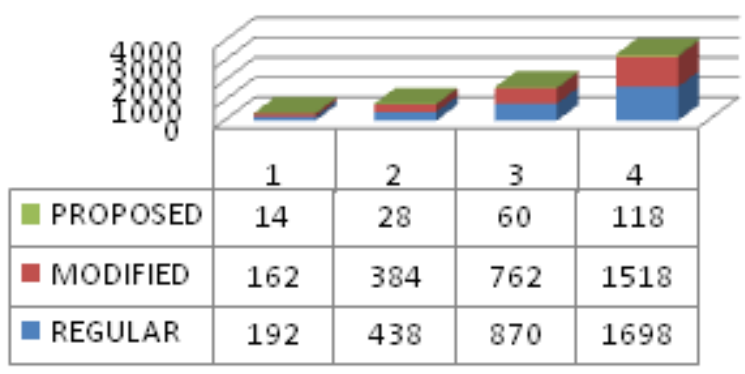

\section{Conclusion}

A regular CSLA uses two copies of the carry evaluation blocks, one with block carry input is zero and other one with block carry input is one. Regular CSLA suffers from the disadvantage of occupying more chip area. The modified CSLA reduces the area and power when compared to regular CSLA with increase in delay by the use of Binary to Excess-1 converter. This paper proposes a scheme which reduces the delay, area and power than regular and modified CSLA by the use of D-latches.

\section{References}

[1] Anitha Kumari R D, Nayana N D-(2011), "Low power and Area Efficient Carry Select Adder", National Conference on Electronics, Communication and Signal Processing, NCECS

[2] 2. Bedrij O.J-(1962), "Carry-select adder," IRE Trans. Electron. Comput., pp.340-344.

[3] Ceiang T Y and Hsiao M J, (Oct. 1998) "Carry-select adder using single ripple carry adder," Electron. Lett., vol. 34, no. 22, pp. 2101-2103,

[4] Jeong.W and Roy .K(2003), "Robust high-performance low power adder", Proc. of the Asia and South Pacific Design Automation Conference, pp. 503-506.

[5] He Y, Chang C H, and Gu J(2005), "An area efficient 64-bit square root carry select adder for low power applications," in Proc. IEEE Int. Symp. Circuits Syst., vol. 4, pp. 4082-4085.

[6] Hosseinghadiry M, Mohammadi H and Nadisenejani M,(2009) " Two New Low Power High Performance Full Adders with Minimum Gates", World Academy of Science, Engineering and Technology 52 .

[7] KeivanNavi and NedaKhandel, (2008)“The Design of a High-Performance Full Adder Cell by Combining Common Digital Gates and Majority Function", European Journal of Scientific Research, ISSN 1450-216X Vol.23 No.4 pp.626-638.

[8] Kim Y and Kim L S(May 2001), "64-bit carry-select adder with reduced area”, Electron.Lett., vol. 37, no. 10, pp. 614-615,

[9] Manoj Kumar, Sandeep K. Arya and SujataPandey, ( December 2011) "Single bit full adder design using 8 transistors with novel 3 transistors XNOR gate", International Journal of VLSI design \& Communication Systems (VLSICS) Vol.2, No.4,.

[10] Massimo Alioto and Gaetano Palumbo, (August 28-31, 2001,)"Optimized Design of Carry-Bypass Adders", ECCTD’01 - European Conference on Circuit Theory and Design, Espoo, Finland.

[11] Padma Devi, Ashima Girdher and Balwinder Singh, (June 2010)"'Improved Carry Select Adder with Reduced Area and Low Power Consumption", International Journal of Computer Applications (0975 - 8887), Volume 3 -No.4,.

[12] Ram Kumar .B and Kittur H.M, (February 2012)“Low-Power and Area-Efficient Carry Select Adder", IEEE transactions on very large scale integration (VLSI) systems, vol. 20, no. 2,

[13] Saiful Islam Md, Muhammad MahbuburRahman, Zerina begum and Mohd.Zulfiquar Hafiz, (2009)"Fault Tolerant Reversible Logic Synthesis: Carry Look-Ahead and Carry-Skip Adders", ACTEA 2009July 15-17, ZoukMosbeh, Lebanon. 